\title{
CARDIOVERSION OF ATRIAL FIBRILLATION
}

\author{
Gregory Y H Lip, Robert D S Watson, Shyam P Singh
}

\section{Suitability for cardioversion}

- Recent onset atrial fibrillation

- No structural heart disease, such as mitral valve disease, poor left ventricular function, dilated left atrium

- Successful treatment of any precipitating cause of atrial fibrillation-for example, thyrotoxicosis, chest infection
Cardioversion from atrial fibrillation to sinus rhythm should be considered for suitable patients as an alternative to leaving the patient in a cardiac arrhythmia and treating with drugs. The potential benefits of a return to sinus rhythm are an improvement in wellbeing and exercise capacity; the avoidance of potentially dangerous drug treatment; and a possible reduction in thromboembolic risk.

\section{Electrical cardioversion}

\author{
Methods of cardioversion \\ Electrical \\ - Synchronised external direct current shock \\ - Transoesophageal \\ - Internal \\ Pharmacological \\ - Class I drugs \\ - Class III drugs
}

Electrical cardioversion works by permitting uniform repolarisation and restoring ordered conduction. After the initial asystolic period, the sinoatrial node rapidly resumes its role as cardiac pacemaker, permitting synchronised atrial electrical activity.

External electrical cardioversion with a synchronised direct current shock can be effective in restoring sinus rhythm. The effectiveness of the procedure can range from $20-90 \%$ as the procedure is highly influenced by the underlying aetiology-the highest success rates are seen in patients with atrial fibrillation secondary to hyperthyroidism, while the lowest rates are seen in patients with severe mitral regurgitation.

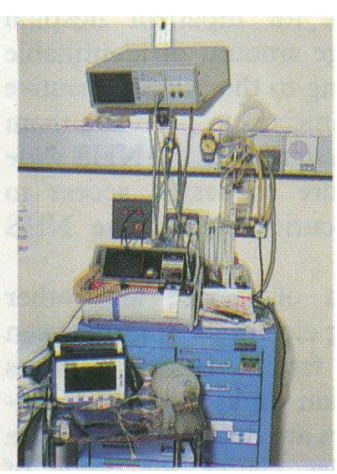

Attention to proper technique for external cardioversion will substantially improve efficacy. The energy requirement and success of external cardioversion are also directly related to the duration of atrial fibrillation, size of the $f$ (fibrillation) waves, and the presence of mitral valve disease (especially if there has been previous valve surgery). Many other factors, however, predict refractoriness to successful cardioversion or unsuccessful maintenance of sinus rhythm.

Transoesophageal cardioversion and internal cardioversion are alternative methods of performing electrical cardioversion, but these are used less often, except in specialist centres.

\section{Pharmacological cardioversion and antiarrhythmic treatment}

In general, class I and III antiarrhythmic drugs are the most useful agents for pharmacological cardioversion
An alternative to electrical cardioversion is pharmacological cardioversion, especially in patients with atrial fibrillation of recent onset. Drugs that are usually used to maintain sinus rhythm after electrical cardioversion are also effective for pharmacological cardioversion. 


\section{Class I agents}

The most commonly used class I drugs are quinidine, flecainide, and propafenone. Quinidine is particularly effective in cardioversion of patients with atrial fibrillation and in maintaining sinus rhythm, but

\section{Procedure for cardioversion}

\section{Preparation}

- Arrange admission for monitoring with electrocardiography-for example, to a coronary care unit

- Ensure electrolytes (especially potassium) are normal

- Ensure anticoagulation is adequate, with an international normalised ratio of 2.0 to $\mathbf{3 . 0}$

- If the patient usually takes digoxin and has no evidence of digoxin toxicity the drug may be taken up to the day before cardioversion; if digoxin toxicity is present then serum digoxin concentrations should be checked and cardioversion delayed

\section{Pharmacological cardioversion}

- Start infusion of drug-for example, flecainide, amiodarone-under continuous monitoring with electrocardiography

Electrical cardioversion

- Arrange for patient to be fasted

- Give short general anaesthetic to eliminate discomfort associated with the transthoracic shock

- Give synchronised direct current shock: start at $100 \mathrm{~J}$, with intermediate "step-ups," eventually to $360 \mathrm{~J}$

- After the procedure monitor the patient for at least one hour to ensure stability of rhythm and blood pressure side effects can occur in a fifth of patients, two thirds of whom have to stop taking the drug.

Flecainide and propafenone have a rate of successful cardioversion of $25-55 \%$ when given orally. Several studies of flecainide have shown it to have a $92 \%$ success rate if given intravenously and to reduce significantly the recurrence of the arrhythmia. The drug has no rate limiting properties, however, and has been reported to cause adverse effects in $74 \%$ of patients. Propafenone, another class Ic compound, may be more useful than flecainide in view of its inherent rate limiting properties (as a $\beta$ blocker), permitting potentially greater ventricular rate control.

\section{Amiodarone}

Amiodarone, a class III antiarrhythmic drug, has been shown to be highly effective in the cardioversion of atrial fibrillation, even in previously refractory cases and in maintaining sinus rhythm. An intravenous infusion of amiodarone acts relatively rapidly, restoring sinus rhythm in up to three quarters of cases, making it as effective as electrical cardioversion. In cases of resistant atrial fibrillation, a four week loading of amiodarone ( $600 \mathrm{mg} /$ day) before cardioversion and a low dose (on average $200 \mathrm{mg} /$ day) maintenance regime after successful cardioversion was effective in achieving cardioversion and sustaining sinus rhythm.

\section{Other drugs}

Although occasionally effective in converting atrial fibrillation to sinus rhythm, verapamil has a much lower success rate of conversion than the class I and III antiarrhythmics. Digoxin is no better than placebo for restoring sinus rhythm. Nor does evidence exist that digoxin is effective as prophylaxis against recurring atrial fibrillation after cardioversion. In addition, in patients with recurrent atrial fibrillation, paroxysms of atrial fibrillation occur more frequently, at a faster rate, and for longer in patients receiving digoxin.

\section{Thromboembolism, antithrombotic treatment, and cardioversion}

\section{Mechanisms and factors contributing to thromboembolism}

\section{Mechanical}

- Sudden resumption of mechanical atrial systole may result in the embolisation of any pre-existing clot, which is dislodged by the mechanical effect of a change in cardiac rhythm during cardioversion

- The return of atrial systole and effective atrial contraction after cardioversion may take up to three weeks

- Cardioversion may promote the formation of new thrombi due to transient atrial dysfunction ("stunning")

\section{Duration of atrial fibrillation}

- A recently formed, poorly adherent thrombus is more likely to dislodge at the time of cardioversion

- The relationship between duration of atrial fibrillation and thromboembolism is affected by haemodynamic status, atrial size, underlying atrial pathology, and effectiveness of anticoagulation

Left atrial size

- Formation of thrombi is more likely in dilated left atria

Abnormalities in haemorheological function and prothrombotic markers

- Clotting factor levels

- Atrial natriuretic peptide, leading to haemoconcentration, and a raised packed cell volume
Peripheral emboli have been estimated to complicate external cardioversion in $1-3 \%$ of cases. Thromboembolism after pharmacological cardioversion probably has similar rates.

The importance of prophylactic anticoagulation in patients with chronic atrial fibrillation is now established. The role of prophylactic anticoagulation to prevent thromboembolism after cardioversion for patients in atrial fibrillation has been clinically examined in several large series, which established that prior anticoagulant treatment was beneficial in attempted cardioversion. The thromboembolic events that occurred in patients who had received anticoagulants were noted predominantly in the first week after cardioversion, suggesting that this is a high risk period.

The dose of warfarin should be adjusted to maintain an international normalised ratio of 2.0 to 3.0 , although in patients at high risk of embolism-for example, those with previous thromboembolism or with mechanical prosthetic heart valves-the target is 3.0 to $4 \cdot 5$. 


\section{Recommendations of American Association of Chest Physicians for anticoagulation before and after cardioversion}

- Warfarin for three weeks before non-emergency cardioversion of atrial fibrillation of $>24-48$ hours' duration

- Warfarin for four weeks after cardioversion

- Intravenous heparin followed by warfarin if cardioversion cannot be postponed for three weeks

- Anticoagulants may not be needed for atrial fibrillation of $<2$ days' duration or for cardioversion of supraventricular tachycardia.

Consideration should be given to managing atrial flutter similarly to atrial fibrillation
After successful cardioversion to sinus rhythm it is important to continue with oral anticoagulants. The routine (and optimal) use of antiarrhythmic drugs before and after cardioversion, however, remains controversial. The use of these drugs after cardioversion is to maintain sinus rhythm and prevent recurrence of arrhythmia.

\section{Predictors of refractoriness to cardioversion or unsuccessful maintenance of sinus rhythm}

\section{- Age $>50$ years}

- Arrhythmia for $>1$ year

- Hypertension

- Structural heart disease, including poor cardiac function, valvar disease, previous mitral valve surgery, and other organic heart disease

- No correctable precipitating factor-for example, thyroid disease, infection
Current practice favours maintaining oral anticoagulation after cardioversion. The risk of embolism probably continues even after successful cardioversion as atrial mechanical function may not be restored for several weeks. The optimal duration of anticoagulation is still unclear, although the American Association of Chest Physicians has drawn up recommendations, including the suggestion that warfarin should be continued for four weeks after cardioversion. In patients with a high risk of recurrent atrial fibrillation, however, it may be prudent to continue anticoagulation for longer than four weeks.

\section{Changes after cardioversion of atrial fibrillation}

\section{Short term}

- Hypotension and bradycardia-bradycardia more common in patients with the sick sinus syndrome and after acute myocardial infarction

- Arrhythmias, usually due to either inadequate synchronisation or digoxin toxicity; ventricular arrhythmias after cardioversion are less common but more serious (ventricular fibrillation is the most common, in about $1 \%$ of cases, but is usually reverted by repeat shock)

- Premature beats and conduction disturbances (first degree or second degree atrioventricular block) are also common

- Small rises in creatine kinase concentration may occur with electrical cardioversion, usually from skeletal muscle, and myocardial damage is unlikely

- Raised transient ST segment after cardioversion, usually associated with previous pericardiotomy, age, and diminished long term maintenance of sinus rhythm

Long term

- Reduction in left atrial size

- Improvement in ventricular function and also in some cases cardiac output and exercise or functional capacity due to a combination of a reduction in heart rate and the restoration of atrial systole
Without antiarrhythmic drugs there is a high risk of relapse of atrial fibrillation, with the proportion of patients remaining in sinus rhythm ranging from $69 \%$ at one month to $58 \%$ at six months, $23 \%$ at one year, and $16 \%$ at two years. These drugs are most useful during the three months after successful cardioversion. If patients have an identifiable cause of atrial fibrillation that has been corrected-for example, thyrotoxicosisantiarrhythmic treatment for three months may be sufficient. If patients, however, have no obvious acute precipitating factors and adverse features for recurrence of atrial fibrillation are present then treatment with antiarrhythmics should be continued for a longer period.

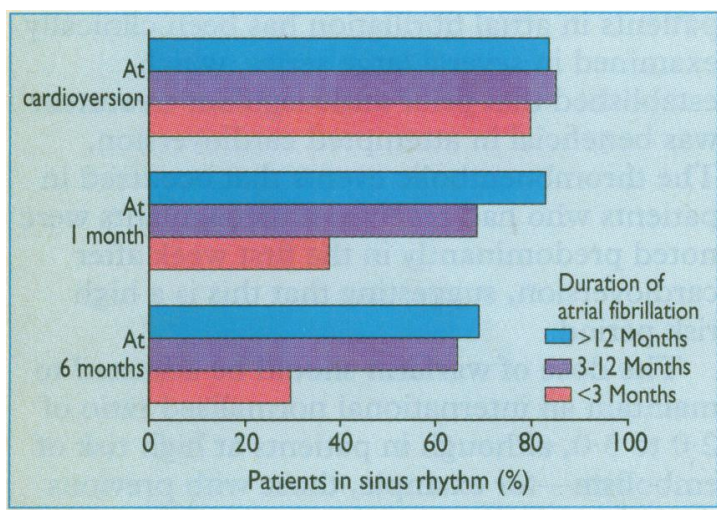

Patients in sinus rhythm at time of cardioversion and at one month and six months of follow up according to duration of atrial fibrillation.

\section{Prognosis after cardioversion}

A long previous duration of arrhythmia, previous episodes of atrial fibrillation, and age $>50$ years predict unsuccessful maintenance of sinus rhythm and reversion to atrial fibrillation. In addition, the presence of coronary artery disease, hypertension, and other organic disease - such as mitral valve disease, aortic stenosis, and cardiomyopathy - are detrimental to maintaining normal sinus rhythm.

Recent studies suggest that left atrial size does not influence the outcome after cardioversion but that the duration of atrial fibrillation is the most important predictor for outcome. Even with a dilated left atrium, long term sinus rhythm (about $80 \%$ at 12 months) is possible with the use of antiarrhythmic drugs. 


\section{Summary}

- Cardioversion to sinus rhythm should be considered for all suitable patients in atrial fibrillation

- In the short term both pharmacological and electrical cardioversion restore sinus rhythm

- Prophylactic treatment with antiarrhythmic drugs is advisable after cardioversion in high risk patients in view of the high relapse rate

- Anticoagulants should be started before non-emergency cardioversionideally two to three weeks before-and continued for at least four weeks after cardioversion in patients with atrial fibrillation of $>48$ hours' duration

\section{Key references}

ACP/ACC/AHA Task Force Statement. Clinical competence in elective direct current (DCO cardioversion. J Am Coll Cardiol 1993:22:336-9

Clark A, Cotter L. Practical procedures-DC cardioversion. Br J Hosp Med 1991;46:114-5

Laupacis A, Albers GW, Dunn JE, Dunn MI, Feinberg W, Jacobson AK. Antithrombotic therapy in atrial fibrillation. Chest 1995;108/suppl0:352-9S Lip GYH. Cardioversion of atrial fibrillation. Postgrad Med J 1995;71:457-65
The duration of the arrhythmia seems to be the most important factor influencing prognosis after cardioversion. Twice as many patients who have had atrial fibrillation for less than three months remain in sinus rhythm as patients who have had atrial fibrillation for more than 12 months.

A more comprehensive version of this article can be found in Lip GYH, Postgrad Med F 1995;71:457-65.

The source of the data in the box of recommendations for anticoagulation before and after cardioversion is Laupacis $A$ et al, Chest 1995;108 (suppl):352-9S. The histogram was adapted from Dittrich et al, Am f Cardiol 1989;63:193-7.

Gregory Y H Lip is a senior registrar and Robert D Watson and Shyam P Singh are consultant cardiologists in the university department of medicine and department of cardiology, City Hospital, Birmingham.

The ABC of Atrial Fibrillation is edited by Gregory Y H Lip, currently in the department of cardiology, Walsgrave Hospital, Coventry.

\title{
Job sharing at a children's hospital: evaluation by medical staff
}

\author{
Jane P Valentine, Cherry J Martin
}

\begin{abstract}
Objective-To evaluate job sharing for registrars at Princess Margaret Hospital for Children, Perth, by seeking responses from members of the relevant medical teams.

Design-A questionnaire was sent to all 126 medical staff within the hospital (and three managers in medical administration) asking their views on job sharing for registrars.
\end{abstract}

Outcome measures-Whether job sharing should continue, who should do it, at what stage of training, and the effects on patient care.

Results-Among the 77 respondents (60\%) there was broad support for the continuation of job sharing at the hospital: only 5 of 37 consultants and 2 of 19 non-job sharing registrars rejected the idea (with a further 4 consultants uncertain). $43 \%$ Of the consultants who had worked with job sharing registrars thought continuity of care was adversely affected.

Conclusion-The committee for physician training of the Royal Australasian College of Physicians emphasises that advanced training should be flexible, with a wide range of opportunities for individuals to plan an appropriate training programme in line with their personal goals. This study has shown that job sharing for registrars at Princess Margaret Hospital for Children allows this choice. Action on concerns over any adverse effects on patient care should resolve any persisting disquiet.

Princess Margaret Hospital, PO Box D184, Perth WA 6001, Australia Jane $P$ Valentine, paediatric registrar

Cherry J Martin, paediatric registrar

Correspondence to: Dr Valentine.

BMF 1996;312:115-6 time they work, and each holds a permanent part time post." When the scheme started job sharing had not been implemented to any significant degree in other teaching hospitals in Australia, ${ }^{1}$ and published reports are confined to sporadic single case reports. ${ }^{3-5}$ The aim of this study was to evaluate job sharing for registrars at Princess Margaret Hospital.

\section{Methods}

A questionnaire was sent to all medical staff at Princess Margaret Hospital and anonymous responses invited. The questionnaire was in a multiple choice format with some questions giving an option for comment. The questions covered five categories of data: (a) details of respondent, gender, and experience of working with a job share team; $(b)$ views on who should be eligible to share jobs; $(c)$ the future role of job sharing for registrars in the hospital; $(d)$ the stage of training most suitable for job sharing; and (e) consultants' views on the impact of job sharing on patient care and supervision and training of registrars. In addition the medical administration department received selected questions on selecting partners for a shared job and the effect or otherwise on their workload.

\section{Results}

Information on the job share team rosters for 1989-93 showed that 53 of the $62(86 \%)$ medical consultants had worked with a job share team at least once. Questionnaire response rates are shown in table 1 . The ratio of the gender of the respondents was in keeping with the known ratio of men to women at each level. All registrars who had taken part in job shares were women and all but one had young children.

All medical groups supported the continuation of job sharing at the hospital (table 2). The responses to the remaining core questions were similar for each group and are presented as an overall response in table 2. Questions about patient care were directed to consultants, and these results are shown in table 3. 